\title{
Clinical decision support is an effective approach to improving the quality of imaging selection in patients with transient ischemic attack or minor stroke
}

\author{
Erik P. Hess ${ }^{1}[1] \cdot$ Karl E. Misulis ${ }^{2}$
}

Received: 22 September 2021 / Accepted: 24 September 2021

() The Author(s), under exclusive licence to Canadian Association of Emergency Physicians (CAEP)/ Association Canadienne de Médecine d'Urgence (ACMU) 2021

Kapoor et al. in their study published in this issue of CJEM [1] describe a quality improvement study to address the challenge of underutilization of CTA of the head and neck for patients at high risk for subsequent stroke. These patients are not ones who clearly qualify for an acute stroke pathway; these are patients who have likely had TIA or minor stroke. While they did not meet criteria for the institution's stroke protocol, they are at significant risk for stroke, so rapid and appropriate workup is indicated. Prior to conducting the study, standard institutional practice for these patients was for ED physicians to order a non-contrast $\mathrm{CT}$ of the head in the ED, arrange outpatient carotid Dopplers within 24-72 h, and send a referral to the stroke prevention clinic. The 2021 AHA/ASA guidelines give some guidance regarding the use of carotid ultrasound for evaluation of TIA. Although carotid ultrasound is listed as one of the diagnostic tests to evaluate for significant stenosis, this guideline, as well as the authors of this editorial, opines that CTA is preferable to carotid ultrasound or MRA [2]. The authors of this study facilitated a meeting of the heads of neurology, radiology, and the ED and identified the following knowledge gaps: the relative value of CTA compared to carotid Doppler ultrasound in the workup of TIA and minor stroke; challenges in identifying patients as high risk; and, concerns about overwhelming radiology resources if CTA of the head and neck became routinely used in this patient population. To address these knowledge gaps, the authors designed and implemented a clinical decision support (CDS) tool in the electronic

Erik P. Hess

erik.hess@vumc.org

1 Department of Emergency Medicine, Vanderbilt University Medical Center, 1313 21st Avenue South, Nashville, TN 37323-4700, USA

2 Department of Neurology, Division of Hospital Neurology, Vanderbilt University Medical Center, Nashville, TN, USA medical record to influence ED physician imaging ordering practices by asking 2 questions: (1) "Does this patient have any persistent neurologic deficits in the ED?"; and, (2) "If deficits have resolved, did the patient have motor and/or speech symptoms within the last $48 \mathrm{~h}$ ?" A positive answer to either of these questions resulted in a CDS prompt, which could be manually overridden, that recommended the ED physician order a CTA of the head and neck. This intervention was employed along with provider education and posting of a memorandum of understanding in the ED declaring support by all three departments. The intervention increased CTA utilization in this patient group from a baseline rate of $12 \%$ to $77 \%$.

Previous publications have supported the findings of this paper that the approach to transient neuro-disturbance is imperfect. This study shows the effectiveness of one approach to the problem. While it was not designed to demonstrate a reduction in subsequent strokes, it does show a potential remedy. A related study reported experience in British Columbia, again indicating that a CDS tool can improve CTA utilization where appropriate, without overwhelming burden on the healthcare system [3].

The authors of this editorial are the directors of Emergency Medicine and Hospital Neurology for a large and very busy academic hospital and comprehensive stroke center. One of us is also an informatics physician. We recognize that efforts such as these will be the way to improve quality of care, reduce overutilization and inappropriate utilization, and ultimately improve outcomes. The authors of this paper are to be applauded for their work and encouraged to continue, with a focus on validation through outcome assessment, and also with continued improvement of their tool and its implementation.

Funding This editorial received no specific grant from any funding agency. 


\section{References}

1. Kapoor, A., Verma, A., Kim, I.J. et al. Multidisciplinary quality improvement initiative to optimize acute neurovascular imaging for transient ischemic attack or minor stroke. Can J Emerg Med. 2021. https://doi.org/10.1007/s43678-021-00180-1.

2. Kleindorfer DO, Towfighi A, Chaturvedi S, Cockroft KM, Gutierrez J, Lombardi-Hill D, Kamel H, Kernan WN, Kittner SJ, Leira EC, Lennon O, Meschia JF, Nguyen TN, Pollak PM, Santangeli P, Sharrief AZ, Smith SC Jr, Turan TN, Williams LS. 2021 Guideline for the prevention of stroke in patients with stroke and transient ischemic attack: a guideline from the
American Heart Association/American Stroke Association. Stroke. 2021;52(7):e364-467. https://doi.org/10.1161/STR.00000 00000000375 (Epub 2021 May 24. Erratum in: Stroke. 2021 Jul; 52(7):e483-e484. PMID: 34024117).

3. Bibok MB, Votova K, Balshaw RF, Penn M, Lesperance ML, Harris DR, Sedgwick C, Nealis M, Farrell B, Mathieson JR, Penn AM. Retrospective evaluation of a clinical decision support tool for effective computed tomography angiography utilization in urgent brain imaging of suspected TIA/minor stroke in the emergency department. CJEM. 2019;21(3):343-51. https://doi.org/10. 1017/cem.2018.449 (Epub 2018 Oct 2 PMID: 30277176). 